\title{
Tofacitinib Decreases Autophagy of Fibroblast-Like Synoviocytes From Rheumatoid Arthritis Patients
}

\author{
M. Vomero ${ }^{1,2}$, M. Caliste ${ }^{3}$, C. Barbati ${ }^{1 *}$, M. Speziali ${ }^{1}$, A. I. Celia ${ }^{1}$, F. Ucci ${ }^{1}$, C. Ciancarella ${ }^{1}$ \\ E. Putro ${ }^{1}$, T. Colasanti ${ }^{1}$, G. Buoncuore ${ }^{1}$, E. Corsiero ${ }^{3}$, M. Bombardieri ${ }^{3}$, F. R. Spinelli ${ }^{1}$, \\ F. Ceccarelli ${ }^{1}$, F. Conti ${ }^{1}$ and C. Alessandri ${ }^{1}$ \\ ${ }^{1}$ Arthritis Center, Dipartimento di Scienze Cliniche Internistiche, Anestesiologiche e Cardiovascolari, Sapienza University of Rome, \\ Rome, Italy, ${ }^{2}$ Rheumatology, Immunology and Clinical Medicine Unit, Università Campus Bio-Medico di Roma, Rome, Italy, \\ ${ }^{3}$ Centre for Experimental Medicine and Rheumatology, William Harvey Research Institute, Queen Mary University of London, \\ London, United Kingdom
}

\section{OPEN ACCESS}

Edited by:

Bo-Zong Shao,

General Hospital of Chinese PLA,

China

Reviewed by:

Yasser M. El-Sherbiny, Nottingham Trent University,

United Kingdom

Srinivasa Reddy Bonam,

Institut National de la Santé et de la Recherche Médicale (INSERM), France

*Correspondence:

C. Barbati

cristiana.barbati@uniroma1.it

Specialty section:

This article was submitted to Inflammation Pharmacology,

a section of the journal

Frontiers in Pharmacology

Received: 11 January 2022

Accepted: 14 February 2022

Published: 03 March 2022

Citation:

Vomero M, Caliste M, Barbati C, Speziali M, Celia Al, Ucci F, Ciancarella C, Putro E, Colasanti T,

Buoncuore G, Corsiero E,

Bombardieri M, Spinelli FR,

Ceccarelli $F$, Conti $F$ and Alessandri $C$

(2022) Tofacitinib Decreases

Autophagy of Fibroblast-Like

Synoviocytes From Rheumatoid Arthritis Patients.

Front. Pharmacol. 13:852802.

doi: 10.3389/fphar.2022.852802
The pathway of Janus tyrosine kinases (JAKs) has a central role in the pathogenesis of Rheumatoid Arthritis (RA) by regulating multiple immune functions and cytokine production. The JAK inhibitor tofacitinib is effective in RA patients not responding to methotrexate or TNF-inhibitors. Since hyperactive autophagy has been associated with impaired apoptosis of RA fibroblast-like synoviocytes (FLS), we aimed to investigate the role of tofacitinib in modulating autophagy and apoptosis in these cells. FLS isolated from RA biopsies were cultured with tofacitinib in presence of autophagy inducer rapamycin and in serum deprivation condition. Levels of autophagy, apoptosis, and citrullinated proteins were analyzed by western blot, flow cytometry, immunocytofluorescence, and Real-Time PCR. Rapamycin induced an increase in RAFLS autophagy while the levels of autophagy marker LC3-II were reduced after in vitro treatment with tofacitinib. The analysis of autophagic flux by specific fluorescence dye confirmed the reduction of autophagy in RA FLS. The treatment with tofacitinib did not influence apoptosis of RA FLS. Modulation of the autophagic process by tofacitinib did not significantly change citrullination. The results of this study demonstrate that tofacitinib is able to modulate autophagy of FLS contributing to its effectiveness in RA patients.

Keywords: Rheumatoid arthritis, tofacitinib, janus tyrosine kinases, autophagy, apoptosis

\section{INTRODUCTION}

Rheumatoid arthritis (RA) is an inflammatory disease characterized by an autoimmune response against self-antigens promoted by adaptive and innate immune cells. RA fibroblast-like synoviocytes (RA-FLS) are subjected to complex molecular changes that lead to an aggressive and invasive phenotype characterized by the release of inflammatory cytokines, chemokines and matrixdegrading enzymes (McInnes and Schett, 2020).

Autophagy is a metabolic process involved in the degradation of intracellular components via lysosomal machinery. Although several autophagy types are described in mammalian cells, macro-autophagy, hereafter referred to as autophagy, is the most studied (Nakatogawa et al., 2009). Autophagy is stimulated by starvation and it is characterized by proteins and organelles engulfment in double-membrane vesicles called autophagosomes. Later, the autophagosome 
fusion with lysosome ensures cargo degradation by lysosomal hydrolases and recycling cytoplasmic material to generate energy. Autophagy-related proteins provide the ongoing process and microtubule-associated protein light chain 3 (LC3) is widely considered to be the most crucial autophagy marker (Klionsky et al., 2021). A dysregulated autophagy has been implicated in the pathogenesis of several inflammatory diseases, including RA (Vomero et al., 2018). In RA-FLS, autophagy is persistently activated leading to chronic inflammation and joint damage. Moreover, RA-FLS showed apoptosis resistance, and increasing evidence supports the hypothesis that defective apoptosis in RA-FLS could result from hyperactivation of autophagy (Shin et al., 2010; Connor at al., 2012). The pathway of Janus tyrosine kinases (JAKs) regulates multiple immune functions and cytokine production involved in the pathogenesis of RA. Tofacitinib is the first generation JAK inhibitor approved by the FDA and EMA for the treatment of RA. The "cross-talk" between JAK/STAT and the stress-activated $\mathrm{PI} 3 \mathrm{~K} / \mathrm{AKT} / \mathrm{mTOR}$ axis, involved in autophagy regulation, seem to be dysregulated in RA (Malemud, 2013). Ireland et al. showed that the addition of an inhibitor of Phosphatidylinositide-3 kinase (PI3K), a downstream step of the JAK pathway, can inhibit the autophagy (Ireland and Unanue, 2011). Nevertheless, there are no other studies on the effect of JAK inhibition on autophagy.

Considering the central role of JAK pathway in the control of RA inflammatory response, we investigated the effects of tofacitinib on autophagy and apoptosis of RA-FLS.

\section{METHODS}

\section{RA-FLS Isolation}

Synovial tissue was drawn during a total knee/hip knee replacement at Queen Mary University of London. After informed consent, samples from five RA patients (patients mean age was 73.25 years and $50 \%$ of patients were female) and from five osteoarthritis (OA) patients, used as control group, were obtained. FLS were purified from synovial tissue after digestion with dispase at $37^{\circ} \mathrm{C}$ for $60 \mathrm{~min}$ as previously described (Vomero et al., 2019). FLS were used between passages four and eight to avoid lymphocytes and macrophages contamination.

\section{Culture Condition and Treatments}

For in vitro experiments, FLS were grow in a Dulbecco's modification of Eagle medium (DMEM) supplemented with $10 \%$ Fetal Bovine serum (FBS, EuroClone) heat inactivated at $50^{\circ} \mathrm{C}$ for $30^{\prime}, 50 \mathrm{IU} / \mathrm{ml}$ penicillin/streptomycin, $2 \mathrm{mM}$ glutamine, and $10 \mathrm{mM}$ HEPES, in a controlled atmosphere incubator at $37^{\circ} \mathrm{C}$. Cells were subjected to different treatments.

- tofacitinib (provided by Pfizer) resuspended in DMSO and then diluted in cell medium at a concentration of $1 \mu \mathrm{M}$;

- rapamycin resuspended in DMSO and then diluted in cell medium at a concentration of $200 \mathrm{nM}$ (Sigma-Aldrich);
- nutrients deprivation (starvation) obtained by culturing cells at a lower serum concentration (2\% FBS).

After $24 \mathrm{~h}$ of culture, FLS were collected for analysis of autophagy and apoptosis as described below. Preliminary experiments on FLS isolated from patients affected by OA were performed (Supplementary Figure S1).

The study was approved by the Ethics Committee of Sapienza University of Rome (protocol number 707/17).

\section{Analysis of Autophagy}

Autophagy levels in FLS were analyzed by evaluating the most common autophagy marker LC3-II by Western blot.

In detail, cells were lysed in RIPA buffer (100 mM Tris-HCL $\mathrm{pH} 8,150 \mathrm{mM} \mathrm{NaCl}, 1 \%$ Triton X-100, $1 \mathrm{mM} \mathrm{MgCl}, 25 \mathrm{mM}$ $\mathrm{NaVO} 4$ and complete protease-inhibitor mixture). After SDSPAGE, western blot was performed using a rabbit anti-human LC3IIB Ab (Cell Signaling) and a rabbit anti-human p62 Ab (Sigma-Aldrich). Peroxidase-conjugated goat anti-rabbit IgG (Bio-Rad) were used as a secondary $\mathrm{Ab}$ and the reaction was developed using the SuperSignal West Pico Chemiluminescent Substrate (Pierce). To ensure the presence of equal amounts of protein a rabbit anti-human $\beta$-actin Ab (Sigma-Aldrich) was used. Quantification of protein expression was performed by densitometry analysis.

Autophagy flux was also measured by flow cytometry by using the Cyto-ID ${ }^{\mathrm{TM}}$ Autophagy Detection Kit (Enzo Life Science) as previously described (Colafrancesco et al., 2020).

Briefly, FLS were pelleted and then resuspended in DMEM without phenol red, supplemented with $10 \%$ FBS, and diluted Cyto-ID solution. After resuspension, the samples were incubated $30 \mathrm{~min}$ at $37^{\circ} \mathrm{C}$ in the dark. After centrifugation, samples were resuspended in assay buffer and transferred into FACS tubes. FACS Calibur (Becton and Dickinson) was employed for all measurement, 50,000 events/sample were run, and data were analysed using the Cell Quest Pro software (BD BioSciences).

For autophagy evaluation by immunocytofluorescence, FLS were cultured on the slides for $24 \mathrm{~h}$ to let them attach and, after treatments, incubated with the anti-human LC3B antibody overnight at $4^{\circ} \mathrm{C}$ (Cell Signaling), with the secondary antibody 30 min (Alexa 555 Goat anti-Rabbit IgG) and finally with DAPI (Sigma-Aldrich) for $15 \mathrm{~min}$. Fluorescence was analyzed by a fluorescence microscope (Olympus BX52). Image acquisition and processing were conducted by IAS 2000 software. MFI has been measured in four different areas for each condition and normalized on the number of cells, as an expression of the number of autophagy vesicles.

\section{Analysis of Apoptosis by Flow Cytometry and Quantitative Real-Time PCR}

The quantitative evaluation of apoptosis was performed after in vitro treatment with tofacitinib using Annexin V (AV) and Propidium iodide (PI) apoptosis detection kit according to the manufacturer's protocol (Marine Biological Laboratory). The reported data were referred to AV-positive apoptotic cells. 


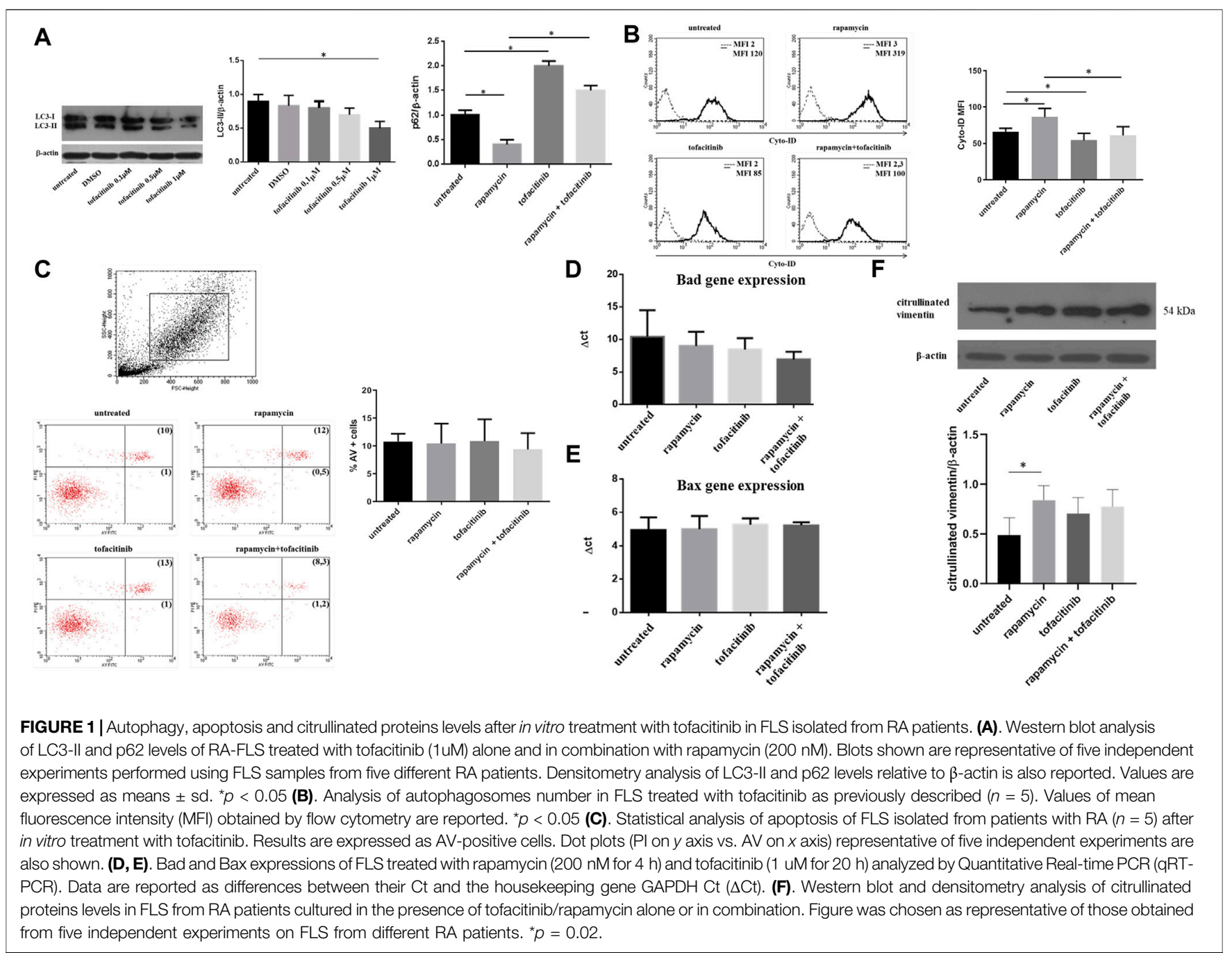

Bad and Bax gene expression was used to quantify apoptosis in qRT-PCR. The RNA was extracted by FLS using the Arcturus Picopure RNA Isolation kit and reverse-transcribed in cDNA by SuperScript IV First-Strand cDNA Synthesis Reaction kit (Thermo Fisher Scientific). For the Real-Time PCR, the cDNA was mixed with dNTPs, Mg++, Taqman probes, and Taq polymerase, and finally amplified in 40 cycles (QuantStudio5 Real-Time PCR System). Probes used are: Bax (Thermo fisher Hs00180269_m1), Bad (Thermo fisher Hs00188930_m1), GAPDH (Thermo fisher Hs02786624_g1). The gene expression has been analyzed in a relative quantification with the $\Delta \mathrm{Ct}$, as the difference between the housekeeping GAPDH cycle threshold (Ct) and the ones of our genes of interest.

\section{Western Blot Analysis of Citrullinated Proteins}

FLS treated with tofacitinib/rapamycin alone or in combination, were lysed in lysis buffer and equal amounts of whole-extract proteins were subjected to $10 \%$ SDS-PAGE. The proteins were blotted onto PVDF membranes (Bio-Rad) and incubated with specific rabbit polyclonal anti-vimentin citrulline $\mathrm{Ab}$ (Millipore). Peroxidase-conjugated goat anti-rabbit IgG (Bio-Rad) were used as a secondary $\mathrm{Ab}$ and the reaction was developed using the SuperSignal West Pico Chemiluminescent Substrate (Pierce). Quantification of protein expression was performed by densitometry analysis.

\section{Statistical Analysis}

Statistical analysis was performed using GraphPad Prism Version 6 (GraphPad Software, San Diego, CA, United States). Data are expressed as mean \pm standard deviation (SD). Data were evaluated using normality test and analyzed using the MannWhitney test. $p<0.05$ was considered to be statistically significant.

\section{RESULTS}

\section{In Vitro Tofacitinib Inhibited Autophagy, but not Apoptosis, of RA-FLS}

First, we investigated whether the addition of the JAKs inhibitor tofacitinib in cell cultures of FLS obtained from RA patients could 

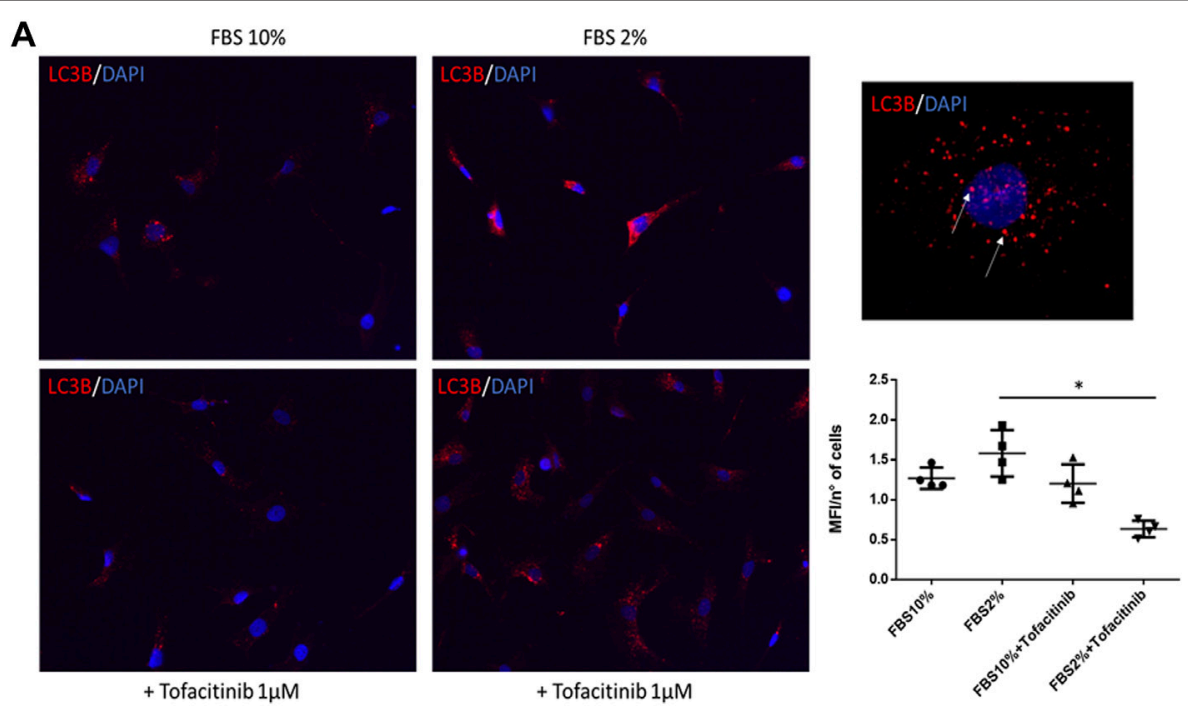

B

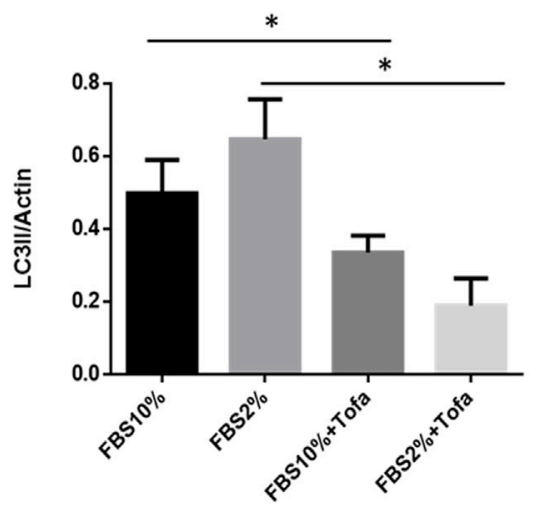

FIGURE 2 Effect of tofacitinib on autophagy in FLS cultured in serum deprivation condition. (A) Immunocytofluorescence analysis of autophagy in FLS treated with tofacitinib alone and in starvation (FBS 2\%). Autophagic LC3B + vesicles are stained in red in RA FLS treated with tofacitinib alone and in serum deprivation condition for $20 \mathrm{~h}(2 \% \mathrm{FBS})$. Cells were counterstained with DAPI dye to reveal DNA (blue staining). Autophagy is expressed as MFI normalized on the number of cells. The quantification was performed on four different areas for each condition. Images are representative of five independent experiments and higher magnification ( $\times 40$ ) picture is also showed. ${ }^{*} p<0.05$. (B) Western blot analysis of autophagy levels in FLS cultured in the presence of tofacitinib. Starvation was used to induce autophagy (FBS $2 \%$ for $20 \mathrm{~h}$ ), and tofacitinib was used as a treatment for $20 \mathrm{~h}(1 \mu \mathrm{M})$. Autophagy was quantified by densitometric analysis on LC3-II and then normalized on $\beta$-Actin. Data are referred to five independent experiments in cells from different RA patients. ${ }^{\star} p<0.05$.

affect autophagy and apoptosis. Based on preliminary doseresponse experiments performed on FLS isolated from OA patients (Supplementary Figure S1), cells were cultured in the presence of tofacitinib at a concentration of $1 \mathrm{uM}$ for $20 \mathrm{~h}$. In vitro experiments were performed using a previous experimental setting consisting in the pre-incubation of FLS with rapamycin to induce autophagy and the subsequent addition of tofacitinib to the culture for $20 \mathrm{~h}$ (Vomero et al., 2019). Western blot analysis showed a reduction of LC3-II after treatment with tofacitinib (Figure 1A). As a confirmation of autophagy inhibition, an accumulation of p62, a selective substrate for autophagy (Ichimura et al., 2008) was also noticed (Figure 1A). The pre-treatment with rapamycin activated autophagy and the subsequent incubation with tofacitinib decreased levels of autophagy marker LC3-II as shown by Western blot analysis (Figure 1A). These results were also confirmed by flow cytometry (Figure 1B). Regarding apoptosis, tofacitinib did not significantly change the percentage of AV-positive FLS (Figure 1C). Also, the expression of the apoptosis-related genes Bad and Bax was not influenced by treatment with tofacitinib (Figures 1D,E). We further analyzed the presence of citrullinated proteins following autophagic stimulus in FLS from patients with RA and with OA. The results revealed a significant increase of citrullinated proteins in cells from RA patients compared with those from OA patients (data not shown). Nevertheless, western blot analysis of RA cells treated with tofacitinib did not show a significant modulation of citrullination compared to untreated cells (Figure 1F). 
Since RA autophagy is involved in FLS survival during stress stimuli, we also evaluated the effect of tofacitinib on autophagy induced by starvation by culturing FLS in serum-deprived medium ( $2 \%$ FBS). As shown in immunocytofluorescence staining, the percentage of autophagic vacuoles was increased in the cells cultured in starvation, and this autophagy induction was prevented by adding tofacitinib (Figure 2A). Furthermore, tofacitinib reduced levels of LC3-II in western blot in FLS cultured in serum deprivation condition (Figure 2B).

\section{DISCUSSION}

This study provides the first evidence that the JAK inhibitor tofacitinib could decrease autophagy of FLS from RA patients. This experimental evidence underlines how autophagy activation is crucial for the survival of inflammatory synoviocytes in RA.

Tofacitinib is a first-generation JAK inhibitor blocking the downstream signal of cytokines whose receptors use JAK3/JAK1 and to a lesser extent JAK2 (Tanaka and Yamaoka, 2013). The precise effect of tofacitinib on different cells involved in RA pathogenesis is largely unknown. In particular, given that the survival of aggressive synoviocytes is considered a key element in the RA pathogenesis, the modulation of FLS autophagy by tofacitinib could explain its therapeutic effects. Recent evidence suggested a role of autophagy in apoptosis decrease of RA synoviocytes and peripheral immune cells (Xu et al., 2013; Vomero et al., 2019). To our knowledge, this is the first study reporting the ability of tofacitinib to downregulate autophagy in synoviocytes from patients affected by RA.

Increased autophagy levels in synovial fibroblasts from RA patients were reported and a possible protective role of autophagy against apoptosis was suggested (Shin et al., 2010). The interaction between JAK/STAT and PI3K/AKT/mTOR axis was involved on the prevention of apoptosis. In fact, it has been demonstrated that the cytokines-induced JAK/STAT pathway contribute to the transcriptional regulation of $\mathrm{Bcl}-2$ family members (Steelman et al., 2008).

Nevertheless, since we have not shown a modulation of apoptosis of RA-FLS, it could be plausible that other signals are needed to activate apoptosis in vitro. This result is partially in agreement with others that showed different proliferation and apoptosis balance of synovial cells in RA patients treated with tofacitinib (Maeshima et al., 2012; Ando et al., 2016).

Citrullination, a chemical conversion of arginine in citrulline by the action of peptidylarginine deiminase enzymes, has a crucial role in RA pathogenesis. In FLS from RA patients, the citrullinated protein levels increased after treatment with the autophagy inducer rapamycin highlighting a possible role of autophagy in the break of self-tolerance (Sorice et al., 2016). In this study, we confirmed this result; however, modulation of the autophagic process by tofacitinib in vitro did not show a significant change in citrullinated proteins. It remains to be clarified whether other post-translational protein modifications could be affected by tofacitinib.
In conclusion, the results of this study elucidated a new mechanism of action of tofacitinib related to autophagy/ apoptosis modulation leading to a better understanding of the possible role of autophagy as a therapeutic target in RA.

\section{DATA AVAILABILITY STATEMENT}

The raw data supporting the conclusion of this article will be made available by the authors, without undue reservation.

\section{ETHICS STATEMENT}

The studies involving human participants were reviewed and approved by the Ethics Committee of Sapienza University of Rome (protocol number 707/17). The patients/participants provided their written informed consent to participate in this study.

\section{AUTHOR CONTRIBUTIONS}

MV designed and performed the experiments, collected the experimental data, examined and interpreted data, and wrote and revised the manuscript. $\mathrm{MC}$ and EC performed immunofluorescence and PCR experiments, performed statistical analysis and revised the article critically. FC (Fulvia Ceccarelli), MS, AIC, FU, CC, FRS helped in data collection and analysis, revised the manuscript. CB, EP and GB performed flow cytometry experiments, examined, analyzed and interpreted data, supervised the work. TC provide support in sample collection. MB patients enrollment and collection of clinical and demographic data. FC (Fabrizio Conti) supervised the work, revised the article. CA conceived the study, wrote the article, and provided financial support for the project. All authors listed have made a substantial, direct, and intellectual contribution to the work and approved it for publication.

\section{FUNDING}

This study was supported by an unconditioned research grant from Pfizer.

\section{SUPPLEMENTARY MATERIAL}

The Supplementary Material for this article can be found online at: https://www.frontiersin.org/articles/10.3389/fphar.2022.852802/ full\#supplementary-material

Supplementary Figure S1 || Dose-response experiments performed on FLS isolated from OA patients. Western blot image of LC3-II protein levels in OA FLS treated with tofacitinib at concentration of $0.1,0.5$ and $1 \mathrm{uM}$ for $20 \mathrm{~h}$. Where indicated, cells were cultured in the presence of DMSO used to resuspend tofacitinib. Densitometry analysis of results obtained from five independent experiments is also shown * $p=0.01$. 


\section{REFERENCES}

Ando, S., Kawada, J. I., Watanabe, T., Suzuki, M., Sato, Y., Torii, Y., et al. (2016). Tofacitinib Induces G1 Cell-Cycle Arrest and Inhibits Tumor Growth in Epstein-Barr Virus-Associated T and Natural Killer Cell Lymphoma Cells. Oncotarget 7, 76793-76805. doi:10.18632/oncotarget.12529

Colafrancesco, S., Vomero, M., Iannizzotto, V., Minniti, A., Barbati, C., Arienzo, F., et al. (2020). Autophagy Occurs in Lymphocytes Infiltrating Sjögren's Syndrome Minor Salivary Glands and Correlates with Histological Severity of Salivary Gland Lesions. Arthritis Res. Ther. 22, 238. doi:10.1186/s13075-020-02317-6

Connor, A. M., Mahomed, N., Gandhi, R., Keystone, E. C., and Berger, S. A. (2012). TNFa Modulates Protein Degradation Pathways in Rheumatoid Arthritis Synovial Fibroblasts. Arthritis Res. Ther. 14, R62. doi:10.1186/ar3778

Ichimura, Y., Kominami, E., Tanaka, K., and Komatsu, M. (2008). Selective Turnover of p62/A170/SQSTM1 by Autophagy. Autophagy 4, 1063-1066. doi:10.4161/auto.6826

Ireland, J. M., and Unanue, E. R. (2011). Autophagy in Antigen-Presenting Cells Results in Presentation of Citrullinated Peptides to CD4 T Cells. J. Exp. Med. 208, 2625-2632. doi:10.1084/jem.20110640

Klionsky, D. J., Abdel-Aziz, A. K., Abdelfatah, S., Abdellatif, M., Abdoli, A., Abel, S., et al. (2021). Guidelines for the Use and Interpretation of Assays for Monitoring Autophagy (4th Edition). Autophagy 17, 1-382. doi:10.1080/15548627.2020. 1797280

Maeshima, K., Yamaoka, K., Kubo, S., Nakano, K., Iwata, S., Saito, K., et al. (2012). The JAK Inhibitor Tofacitinib Regulates Synovitis through Inhibition of Interferon- $\gamma$ and Interleukin-17 Production by Human CD4+ T Cells. Arthritis Rheum. 64, 1790-1798. doi:10.1002/art.34329

Malemud, C. J. (2013). Intracellular Signaling Pathways in Rheumatoid Arthritis. J. Clin. Cel Immunol. 4, 160. doi:10.4172/2155-9899.1000160

McInnes, I. B., and Schett, G. (2011). The Pathogenesis of Rheumatoid Arthritis. N. Engl. J. Med. 365, 2205-2219. doi:10.1056/NEJMra1004965

Nakatogawa, H., Suzuki, K., Kamada, Y., and Ohsumi, Y. (2009). Dynamics and Diversity in Autophagy Mechanisms: Lessons from Yeast. Nat. Rev. Mol. Cel Biol. 10, 458-467. doi:10.1038/nrm2708

Shin, Y. J., Han, S. H., Kim, D. S., Lee, G. H., Yoo, W. H., Kang, Y. M., et al. (2010). Autophagy Induction and CHOP Under-expression Promotes Survival of Fibroblasts from Rheumatoid Arthritis Patients under Endoplasmic Reticulum Stress. Arthritis Res. Ther. 12, R19. doi:10.1186/ar2921

Sorice, M., Iannuccelli, C., Manganelli, V., Capozzi, A., Alessandri, C., Lococo, E., et al. (2016). Autophagy Generates Citrullinated Peptides in Human
Synoviocytes: a Possible Trigger for Anti-citrullinated Peptide Antibodies. Rheumatology (Oxford) 55, 1374-1385. doi:10.1093/rheumatology/kew178

Steelman, L. S., Abrams, S. L., Whelan, J., Bertrand, F. E., Ludwig, D. E., Bäsecke, J., et al. (2008). Contributions of the Raf/MEK/ERK, PI3K/PTEN/Akt/mTOR and Jak/STAT Pathways to Leukemia. Leukemia 22, 686-707. doi:10.1038/leu. 2008.26

Tanaka, Y., and Yamaoka, K. (2013). JAK Inhibitor Tofacitinib for Treating Rheumatoid Arthritis: from Basic to Clinical. Mod. Rheumatol. 23, 415-424. doi:10.1007/s10165-012-0799-2

Vomero, M., Barbati, C., Colasanti, T., Perricone, C., Novelli, L., Ceccarelli, F., et al. (2018). Autophagy and Rheumatoid Arthritis: Current Knowledges and Future Perspectives. Front. Immunol. 9, 1577. doi:10.3389/fimmu. 2018.01577

Vomero, M., Manganelli, V., Barbati, C., Colasanti, T., Capozzi, A., Finucci, A., et al. (2019). Reduction of Autophagy and Increase in Apoptosis Correlates with a Favorable Clinical Outcome in Patients with Rheumatoid Arthritis Treated with Anti-TNF Drugs. Arthritis Res. Ther. 21, 39. doi:10.1186/s13075-0191818-x

Xu, K., Xu, P., Yao, J. F., Zhang, Y. G., Hou, W. K., and Lu, S. M. (2013). Reduced Apoptosis Correlates with Enhanced Autophagy in Synovial Tissues of Rheumatoid Arthritis. Inflamm. Res. 62, 229-237. doi:10.1007/s00011-0120572-1

Conflict of Interest: The authors declare that the research was conducted in the absence of any commercial or financial relationships that could be construed as a potential conflict of interest.

Publisher's Note: All claims expressed in this article are solely those of the authors and do not necessarily represent those of their affiliated organizations, or those of the publisher, the editors and the reviewers. Any product that may be evaluated in this article, or claim that may be made by its manufacturer, is not guaranteed or endorsed by the publisher.

Copyright (C) 2022 Vomero, Caliste, Barbati, Speziali, Celia, Ucci, Ciancarella, Putro, Colasanti, Buoncuore, Corsiero, Bombardieri, Spinelli, Ceccarelli, Conti and Alessandri. This is an open-access article distributed under the terms of the Creative Commons Attribution License (CC BY). The use, distribution or reproduction in other forums is permitted, provided the original author(s) and the copyright owner(s) are credited and that the original publication in this journal is cited, in accordance with accepted academic practice. No use, distribution or reproduction is permitted which does not comply with these terms. 